\title{
Assessing the quality of ionogram interpretation using the HF Doppler technique
}

\author{
D. Buresova $^{1}$, V. Krasnov ${ }^{1,2}$, Ya. Drobzheva ${ }^{1,2}$, J. Lastovicka ${ }^{1}$, J. Chum ${ }^{1}$, and F. Hruska ${ }^{1}$ \\ ${ }^{1}$ Institute of Atmospheric Physics, 14131 Prague, Czech Republic \\ ${ }^{2}$ Institute of the Ionosphere, Almaty, Republic of Kazakhstan
}

Received: 22 December 2005 - Revised: 13 February 2007 - Accepted: 30 March 2007 - Published: 8 May 2007

\begin{abstract}
The first joint common volume measurements by the Digisonde Portable Sounder (DPS-4) and a new Doppler type system has been run at the Pruhonice ionospheric observatory $\left(49.99^{\circ} \mathrm{N}, 14.54^{\circ} \mathrm{E}\right)$ since January 2004 . The measurement of the Doppler shift is carried out continuously on a frequency of $3.6 \mathrm{MHz}$, thus the radio wave is reflected predominantly from the ionospheric $\mathrm{F}$ layer. To compare digisonde measurements with the Doppler data, a phase path was calculated from both Doppler and digisonde records. Under stormy conditions and in the case where a sporadic E layer was present, a significant disagreement between both measurements has been found. The discrepancies could be related to the uncertainties of the observational inputs and to the interpretation of the digisonde data. The comparison of the phase paths shows that during geomagnetically quiet days, in the absence of the sporadic E layer, and when high quality ionograms are available and correctly scaled, the electron density N(h) profiles, calculated by the Automatic Real Time Ionogram Scaler with True height algorithm (ARTIST), can be considered reliable.
\end{abstract}

Keywords. Ionosphere (Mid-latitude ionosphere; Instruments and techniques)

\section{Introduction}

The quality of ionospheric radio communication depends critically on space weather conditions, and particularly on the state of the ionospheric ionisation. Important ionospheric information is obtained through vertical incidence sounding. Presently, a global network of modern groundbased ionosondes supplies users with real-time automatically scaled ionospheric parameters. Due to the growing need of real-time mapping and short-term predictions, the qual-

Correspondence to: D. Buresova

(buresd@ufa.cas.cz) ity of the ionosonde measurement interpretation becomes more and more important. Over the past decades many research teams have sought solutions to the complicated task of replacing manual ionogram interpretation with automated computer techniques and improving the reliability of scaled ionospheric parameters, making the data, in combination with models, more useful for nowcasting of ionospheric conditions (e.g. Galkin and Dvinskikh, 1968; Wright et al., 1972; Reinisch and Huang, 1983; Titheridge, 1986; Bossy, 1994; Chen et al., 1994; Bibl, 1998; Pezzopane and Scottto, 2005; Reinisch et al., 2005). Nevertheless, the following uncertainties are still possible during ionospheric vertical sounding:

- uncertainties of the ionosonde measurements themselves,

- uncertainties due to incorrect auto scaling (large gaps in ionogram traces, gaps produced by strong interference, insufficient quality of the pattern recognition of the tracing algorithm itself) (Reinisch et al., 2005; Pezzone and Scotto, 2005),

- limitation of the profile inversion algorithm (uncertainties near the critical frequencies; uncertainties due to the presence of the sporadic E layer (Paul, 1986); ionosonde cannot directly determine the electron density profile in the valley between the $\mathrm{E}$ and $\mathrm{F}$ layers (where it uses the valley model and adjusts parameters so as to match the measured F-trace, Reinisch and Huang, 1983).

It is difficult to take into account all these uncertainties and estimate a confidence interval for the resulting data. However, it is possible to test the quality of the experimental results by their comparison with data obtained by another type of apparatus. In particular, ionospheric disturbances well appear on Doppler records. The uncertainty within the Doppler shift measurement is mainly determined by the instability of the frequency of reference generators and errors arising due to interference of radio waves. Both errors can be reduced

Published by Copernicus GmbH on behalf of the European Geosciences Union. 
with the help of apparatus design and corresponding computer programs. The Doppler shift of a radio wave can be calculated using the equation (Davies, 1969):

$f_{d}=-\frac{f}{c_{L}} \frac{d P}{d t}$,

where $f$ is the carrier frequency of the radio sounder, and $c_{L}$ is the speed of light,

$P=\int_{s} n \cos \alpha d s$

is the phase path of a radio wave, $\alpha$ is the angle between trajectory of radio ray and $\mathrm{z}$ axis (z is vertical coordinate), $s$ is the path of radio ray from transmitter to receiver and $n$ is the refractive index : $n=\sqrt{1-A}$, where (Davies, 1969):

$A=\frac{2 w(1-w)}{2(1-w)-u \sin ^{2} \lambda \pm \sqrt{u^{2} \sin ^{4} \lambda+4 u(1-w)^{2} \cos ^{2} \lambda}}$

$w=N / N_{m}, \quad N_{m}=1.24 f^{2} 10^{10}$ electron $/ \mathrm{m}^{3}$ is the electron density at the height of radio wave reflection, $N$ is the electron density at a given height, $u=f_{H}^{2} / f^{2}, \lambda$ is the angle between direction of the geomagnetic field and radio wave trajectory, $f_{H}$ is the gyro frequency for geomagnetic latitude of ionospheric sounding, the sign "+" is for ordinary waves and "-" is for extraordinary waves. The absorption of radio waves and the variation of geomagnetic field with time have been neglected.

Equation (2) takes into account the electron density profile $(\mathrm{N}(\mathrm{h})$ profile) from the initial height to the height of radio wave reflection. To compare the digisonde and Doppler data we use $\mathrm{N}(\mathrm{h})$ profiles obtained from ionograms by the inversion algorithm NHPC (a program for inversion of scaled ionogram traces into electron density profiles) incorporated in the ARTIST scaling program (Automatic Real Time Ionogram Scaler with True height algorithm) (Reinisch and Huang, 1983). The inversion technique is based on the least squares fitting of modified Chebyshev polynomials to the profiles of each of the ionospheric layers. The method of ionospheric $\mathrm{F}$ region botomside ionogram processing is described in details by Reinisch and Huang (1983). Then we calculate the phase path $P$ (Eq. 2) of the sounding radio wave, $P_{i}$. To calculate the phase path $P_{d}$ on the basis of Doppler record, we use an equation derived from Eq. (1):

$P_{d}=-\frac{c}{f} \int_{0}^{t} f_{d}(t) d t+h_{0}$,

where $h_{0}$ is a constant.

The relative accuracy of $P_{d}$ is determined only by the accuracy of measurement of the Doppler shift $f_{d}$, while the absolute accuracy is influenced also by the accuracy of $h_{0}$ determination. Parameter $h_{0}$ in Eq. (4) cannot be directly calculated from Doppler records. However, we can calculate the entire phase path using $\mathrm{N}(\mathrm{h})$ profiles inverted from the recorded ionograms and Eq. (2) - the inversion method considers the ordinary trace (O-trace). For the given day we select the intervals, when there is only an O-trace on the ionograms in the surrounding of the working frequency of the Doppler measurements. Thus, we know that we have only the O-trace on the Doppler records. Then we assume for these intervals that the phase paths obtained from the ionogram $\left(P_{i}\right)$ and from the Doppler measurement $\left(P_{d}\right)$ are equal. For the given day we obtain several data points, which can provide slightly different $h_{0}$. Then we fit the set of individual $P_{i}$ and $P_{d}$, using the least squares method to obtain the value of $h_{0}$. We assume that the phase path from the ground surface to the radio wave reflection point equals to the backward phase path from the radio wave reflection point to the ground surface. Therefore, for comparison of the phase paths we use $P_{c}(t)=P_{i}(t) / 2$ and $P_{e}(t)=P_{d}(t) / 2$; time variations of $P_{c}$ and $P_{e}$ are similar to time variations of the reflection height.

The aim of the study is to verify the ionogram interpretation quality by comparing phase paths computed from digisonde-derived N(h) profiles and Doppler apparatus measurements during simultaneous common volume measurements.

\section{Doppler apparatus}

The Doppler apparatus has been designed and constructed in the Institute of Atmospheric Physics (IAP), Prague. The measurement is carried out at a working frequency of about 3.6 MHz (3.5945 MHz). Therefore the radio wave is reflected predominantly from the ionospheric $\mathrm{F}$ region. The frequency of the transmitter, as well as the receiver frequency, is derived from the $10 \mathrm{MHz}$ reference oscillators by means of direct digital synthesis. The short time stability of the oscillators is $2 \cdot 10^{-10} \mathrm{f}$. The output power of the transmitter was lowered to $1 \mathrm{~W}$ to avoid interference with the other measurements, while the signal-to-noise ratio showed only negligible degradation. The transmitting antenna in use is a small $5 \times 3 \mathrm{~m}^{2}$ magnetic loop, whereas at the receiving side, only a simple $\lambda / 4$ wire is used. The receiver is built on a "zero IF" direct conversion scheme with $80 \mathrm{~Hz}$ AF pitch. The I-Q outputs of the receiver (two signals in quadrature) are fed to the two-channel synchronous AD converter and data stored via local area network in the selected computer. The final signal (signal corresponding to one sideband) is obtained by digital signal processing in the frequency domain.

The transmitter is located at the Pruhonice observatory $\left(49.99^{\circ} \mathrm{N}, 14.54^{\circ} \mathrm{E}\right)$, which is only about $7 \mathrm{~km}$ from the receiver located in Prague at the main building of the Institute. Thus we obtain the Doppler shifted signal nearly vertically reflected. A great advantage of this topological arrangement is the simultaneous operation and common volume measurements with the digital ionosonde (Digital Portable Sounder DPS-4) located also at Pruhonice, which turned out to be very helpful in the interpretation of data. The drawback of 

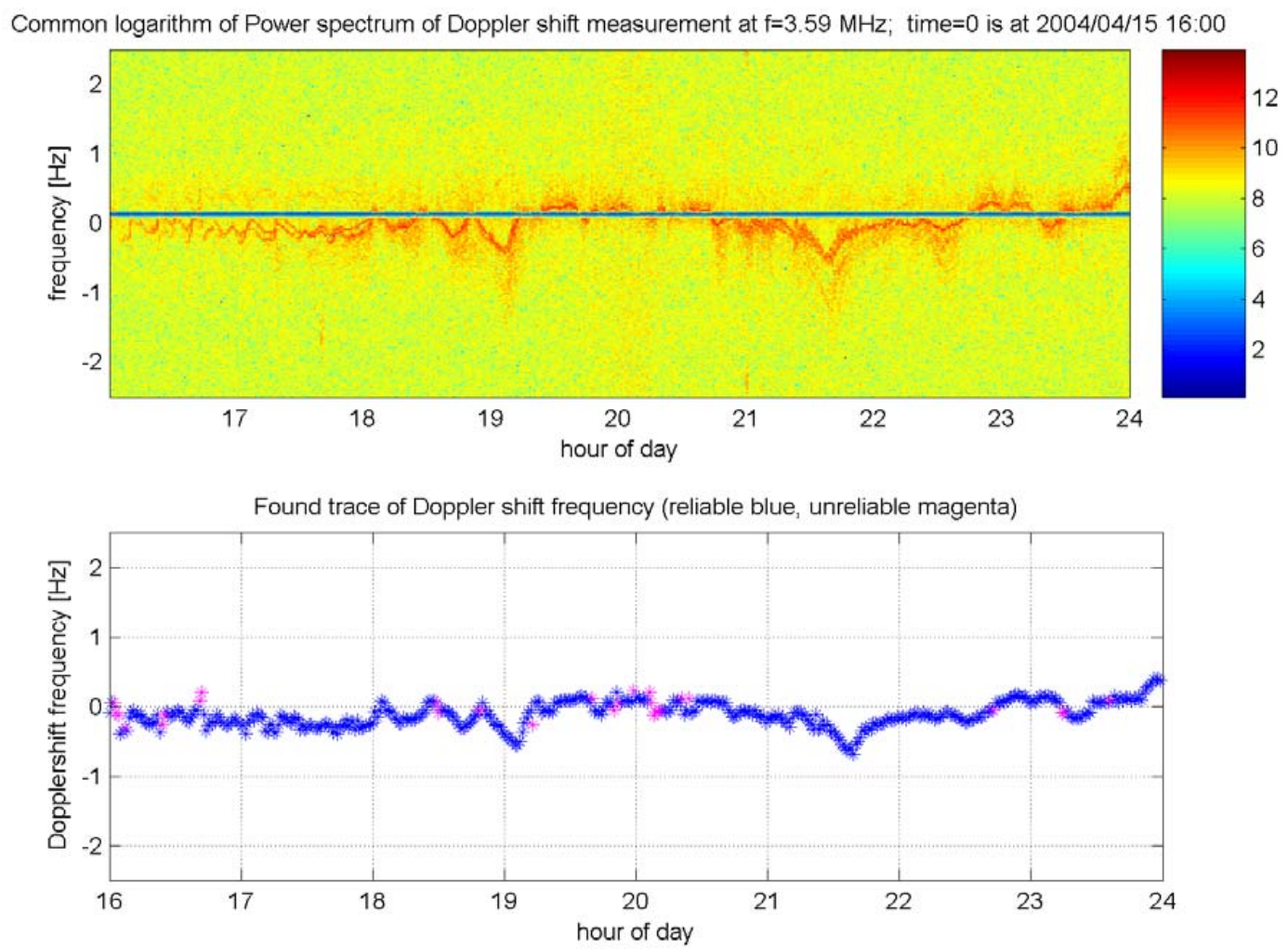

Fig. 1. An example of a Doppler record: the upper plot is the initial Doppler spectrogram, the lower plot represents a curve of Doppler frequency shift corresponding to the spectral peak in the spectrogram found by special software. Blue colour depicts points of high reliability; the magenta colour depicts points of low reliability. Time is in UT.

this arrangement is a strong ground wave that makes the detection of small Doppler shift ( less than $0.04 \mathrm{~Hz}$ ) impossible. We eliminate the signal corresponding to the ground wave by digital processing in the frequency domain. On the other hand, the ground wave provides us with direct verification of the stability of the oscillators and zero drift line.

Since we are using a frequency in the amateur band, we have to transmit a call sign each minute. The duration of the call sign is $\sim 5 \mathrm{~s}$. During that time the data acquisition is stopped. That means that the maximum time interval that can be processed by the FFT algorithm is $\sim 55 \mathrm{~s}$, so the best frequency resolution that we can obtain is $\sim 1 / 55=0.018 \mathrm{~Hz}$. The receiver and transmitter are synchronised by GPS clock. The data are visualised by means of spectrograms, usually with a time resolution of $1 \mathrm{~min}$.

The Doppler system has one more working frequency near 7.0 MHz. However, there are technical problems with high level of noise at this frequency and, moreover, it is suitable only during daytime and moderate and high solar activity conditions, not near the solar cycle minimum like in 2006, when foF 2 is almost all the time below $7 \mathrm{MHz}$.

\section{Comparison of phase paths calculated from iono- grams and Doppler records}

A routine ionosonde measurement is usually repeated once every $15 \mathrm{~min}$. To compare ionogram and Doppler measurements we selected 21 days, when there was only one trace or one trace clearly dominated on Doppler records (high quality data). As a result, we could detect a spectral peak and follow this peak from spectrum to spectrum. To present a detailed analysis of each of the 21 individual days would be boring for readers. Therefore, we present the comparative analysis only for nine representative days $(41,42,68,69,93,96,106,117$, 149) of 2004. Figure 1 shows an example of such Doppler records during day 106 of 2004.

As mentioned above, routine $\mathrm{N}(\mathrm{h})$ profiles are processed from recorded ionograms automatically by the ARTIST software. However, the automatic scaling can sometimes be incorrect. Thus, we have manually tested and, when necessary, corrected the automatic scaling of all ionograms involved in this study. Phase paths have been computed using only N(h) profiles obtained from manually revised ionogram scaling. On the other hand, ionograms shown in the paper (Figs. 2 and 5-8) are digisonde-generated automatic scaling figures, used solely to detect intervals, when only ordinary trace, or 


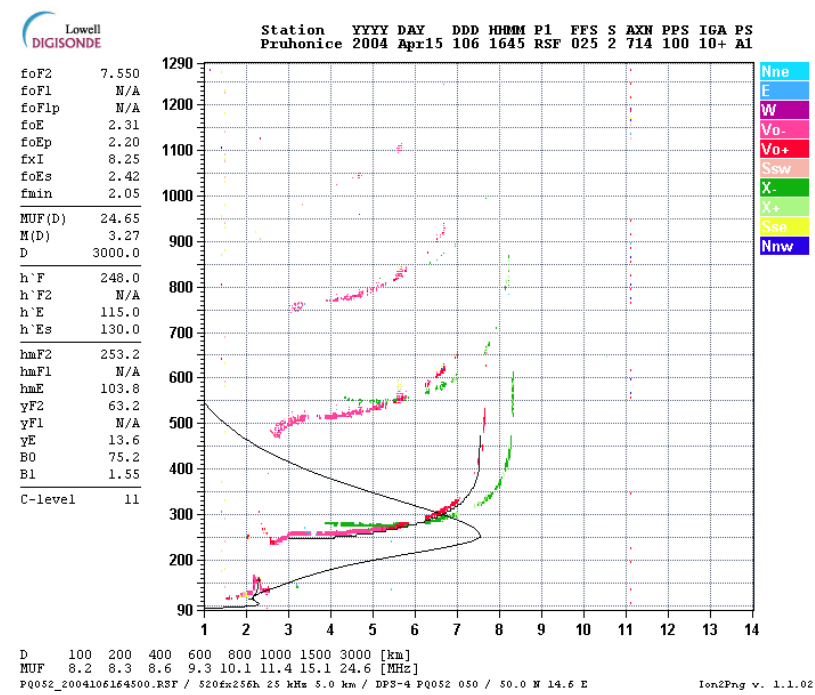

Fig. 2. An example of the ionogram recorded at Pruhonice at 16:45 UT of the day 106 of 2004, when only the O-trace marked by red colour was present in the vicinity of $\mathrm{f}=3.6 \mathrm{MHz}$.

only extraordinary trace, or sporadic E layer are present at ionograms.

There is an ambiguity in Doppler measurements since we are not sure what mode of wave (ordinary or extraordinary) we are observing on the Doppler records. To remove this ambiguity, we used the following approach:

1. We consider the presence or absence of $\mathrm{O}$ - (ordinary) and $\mathrm{X}$ - (extraordinary) echo traces near $3.6 \mathrm{MHz}$ on ionograms. The absence of X-trace (O-trace) on ionograms (see an example of such a case in Fig. 2) during some intervals allows us to claim that the Doppler system measures the $\mathrm{O}$ - trace (X-trace), that trace which is present at ionograms.

2. In the case where both $\mathrm{O}$ - and $\mathrm{X}$-traces are present close to $3.6 \mathrm{MHz}$, we calculate $P_{c}(\mathrm{t})$ (the phase path, calculated from the ionogram) for O-trace and for X-trace and compare the calculated phase paths with the experimental phase path $P_{e}(\mathrm{t})$ obtained from Doppler measurements for every interval. We calculate the average difference $(m)$ based on squares of individual differences between calculated and experimental phase paths. We use $m$ to define what components, O- or X-echo traces, coincide in the best way with experimental one, and we suppose that this mode of wave is what we observe on the Doppler records.

Figure 2 displays a gap in the record at $3.6 \mathrm{MHz}$ as a consequence of interference with the Doppler system signal during common volume measurements. However, presence of only the $\mathrm{O}$ trace on ionogram at frequencies below and above the gap allows us to assume that only the $\mathrm{O}$ trace is present at
3.6 MHz. Similar gaps at $3.6 \mathrm{MHz}$ are observed also at other ionograms shown in the paper - always for the same reason and without impact on our considerations and results.

Figures 3 and 4 present examples of typical results of comparison of the calculated and experimental phase paths.

\subsection{Day 149 of 2004 (Fig. 3a)}

We observed only the O-trace on the ionograms in the region nearby to $3.6 \mathrm{MHz}$ from 04:45 to 05:00 UT and then from $15: 45$ to $17: 00 \mathrm{UT}$. We used these intervals to determine $h_{0}$. A sporadic E-layer $\left(\mathrm{E}_{S}\right)$ was present on the ionograms from 05:15 to 12:30 UT. Both O- and X-traces were recorded on the ionograms in the region close to $3.6 \mathrm{MHz}$ from 00:15 to 04:45 UT. Comparing with the experimental phase path, the difference for the calculated phase paths for O-wave was $m=7.6 \mathrm{~km}$, while for X-wave $m=18.2 \mathrm{~km}$. Thus, $P_{c}(t)$ of $\mathrm{O}$-wave agrees better with the experiment. The average difference $m$ is calculated as an average value from absolute values of differences (i.e. irrespective of their sign) between ionogram and Doppler phase paths. Thus small $m$ means good coincidence between phase paths. There is a sharp shift of the experimental phase path from 05:00 to 05:15 UT, because the radio wave reflection point dropped from the F-layer to $\mathrm{E}_{s}$. There was an $\mathrm{O}$-echo trace and $\mathrm{E}_{s^{-}}$ trace on the ionograms close to $3.6 \mathrm{MHz}$ from 12:45 to $15: 30$. The comparison of $P_{c}(\mathrm{t})$ with $P_{e}(\mathrm{t})$ shows $m=8.2 \mathrm{~km}$ for $\mathrm{O}$ trace and $m=5.4 \mathrm{~km}$ for the $\mathrm{E}_{s}$-trace in this interval. Thus, $P_{c}(t)$ for the wave reflected from the $\mathrm{E}_{s}$ layer agrees better with the experiment. Another sharp shift of the experimental phase path was observed from 15:45 to 16:00 UT. In this case the radio wave reflection point jumped from $\mathrm{E}_{S}$ to $\mathrm{F}$ layer. Again, both O- and X-echo traces were present on the ionograms in the vicinity of $3.6 \mathrm{MHz}$ from $17: 15$ to $23: 45 \mathrm{UT}$. The comparison of calculated phase path with experimental one reveals $m=12 \mathrm{~km}$ for O-trace and $m=5.5 \mathrm{~km}$ for X-trace. Thus, we consider $P_{c}(t)$ of $\mathrm{X}$-trace to be closer to the experimental phase path. ARTIST does not take into account the sporadic E layer when computing electron density profile. Sporadic E layer was present on the ionograms from 05:15 to 15:30 UT, as illustrates an ionogram and corresponding N(h) profile shown in Fig. 5. As a result, the differences between $P_{c}(t)$ for O-wave and $P_{e}(t)$ was $m=11 \mathrm{~km}$.

\subsection{Day 106 of 2004 (Fig. 3b)}

We saw a few intervals on ionograms, where only the O-trace in the region of $3.6 \mathrm{MHz}$ was present. We used these intervals to define $h_{0}$. The comparison of calculated phase paths with experimental ones shows $m=6.3 \mathrm{~km}$ and $m=22.8 \mathrm{~km}$ for O-trace and X-trace, respectively. So, the $P_{c}(\mathrm{t})$ calculated for the O-trace shows better agreement with experimental phase paths $P_{e}(t)$. Some discrepancies between the phase paths in the interval from 11:00 to $16: 00$ UT are caused by sporadic $\mathrm{E}$ or by gaps in the trace on the ionogram (Fig. 6). It 

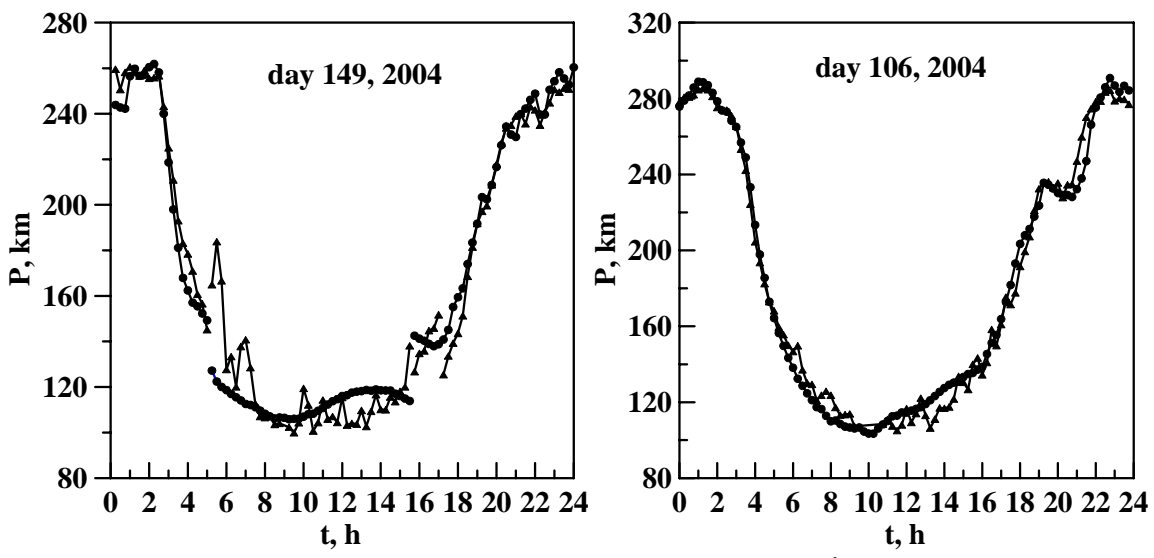

$\mathrm{b}$
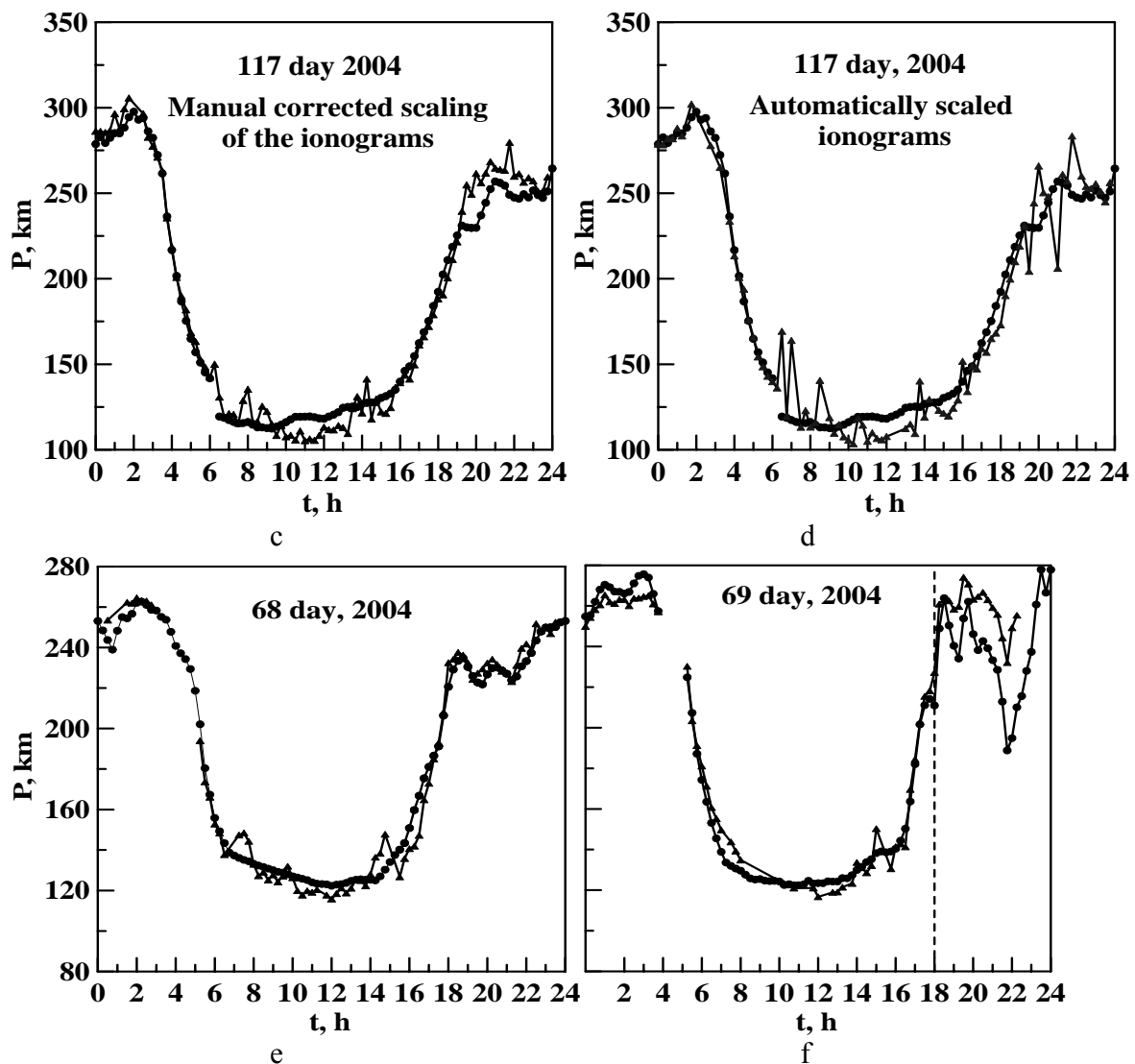

Fig. 3. Typical phase paths of radio wave $(\mathrm{f}=3.6 \mathrm{MHz})$ calculated from ionogram (triangles) and inferred from Doppler records (full-circle lines) for the days 68, 69, 106, 117 and 149 of 2004 involved in the study. Comparison of the $P_{c}(t)$ calculated from manually corrected and automatically scaled ionograms with $P_{e}(t)$ are shown in the plots $(\mathbf{c})$ and $(\mathbf{d})$, respectively. Time is in UT.

is necessary to note that the day 106 was geomagnetically quiet day ( $D_{s t}$ index was smaller than 11$)$, the quality of the recorded ionograms was high, and the manual correction of ionogram scaling practically not necessary.

\subsection{Day 117 of 2004 (Figs. 3c, d)}

On the Pruhonice digisonde records the O-trace dominated close to $3.6 \mathrm{MHz}$ from $05: 45$ to 06:00. Well-developed $\mathrm{E}_{S}$-trace was observed during the intervals from 06:30 to 09:45 UT, from 12:45 to 14:30 UT, from 15:00 to 15:30 UT, and from 19:00 to 22:30. Both O- and X-echo traces were 

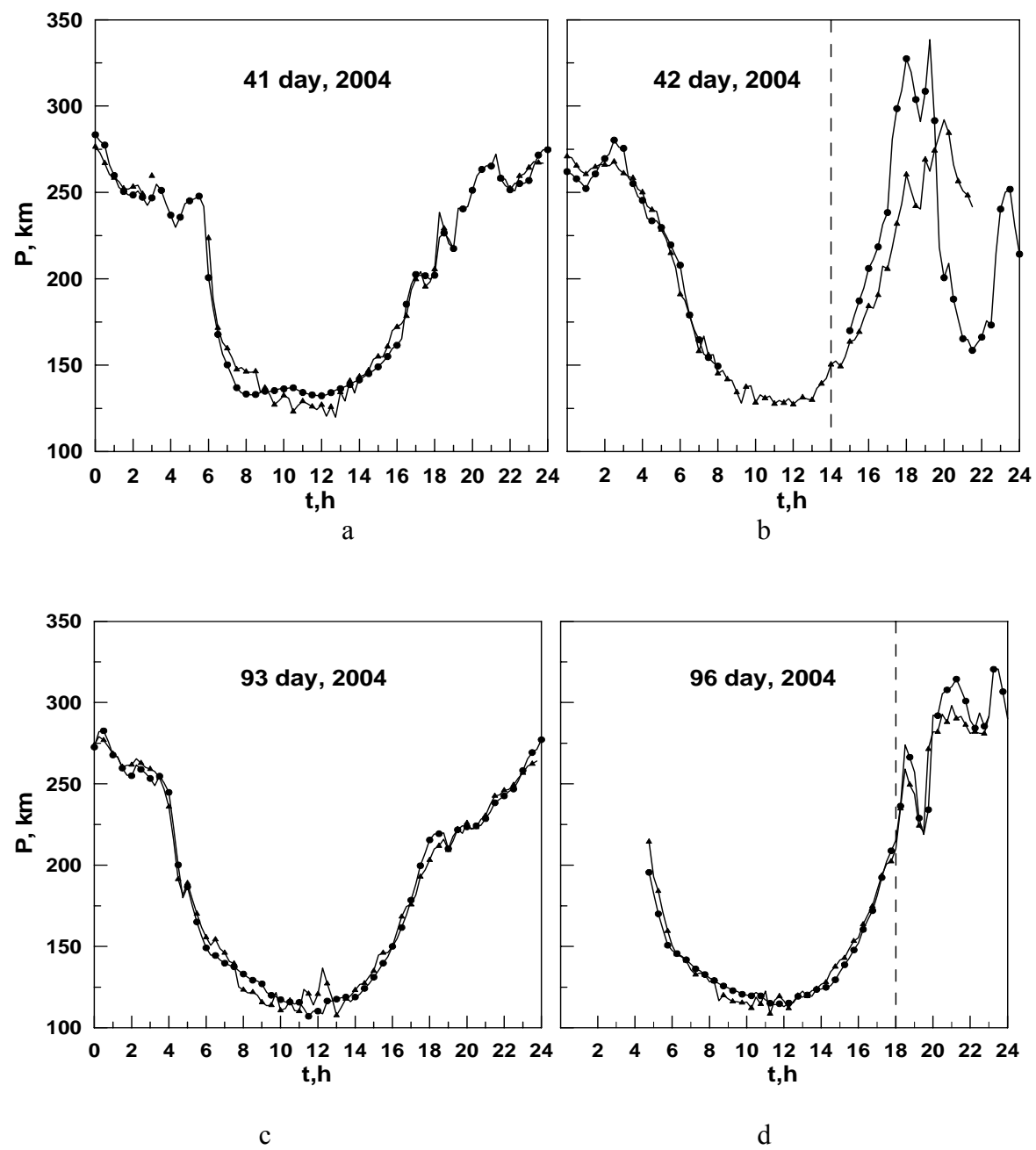

Fig. 4. Typical phase paths of radio wave ( $\mathrm{f}=3.6 \mathrm{MHz}$ ) calculated from ionogram (triangles) and inferred from Doppler records (full-circle lines) for the days 41, 42, 93 and 96 of 2004 involved in the study. Time is in UT.

present on the ionograms close to $3.6 \mathrm{MHz}$ from midnight to 05:30 UT. Comparing with $P_{e}(t)$, we obtained $m=2.56 \mathrm{~km}$ for $P_{c}(t)$ calculated for O-trace, and $m=33.6 \mathrm{~km}$ for the phase path calculated for $\mathrm{X}$-trace within this time interval. Thus, $P_{c}(t)$ of O-trace agrees better with the experimental $P_{e}(t)$. There is an altitudinal significant shift of the experimental phase path from 06:15 to 06:30 UT (Fig. 3c), probably caused by the movement of the radio wave reflection height from $\mathrm{F}$ layer to the $\mathrm{E}_{s}$ layer. There were both ordinary and extraordinary $\mathrm{F}$ layer traces and the trace of $\mathrm{E}_{s}$ layer on the ionograms close to $3.6 \mathrm{MHz}$ from $15: 45$ to $16: 15 \mathrm{UT}$. The comparison of $P_{c}(t)$ with $P_{e}(t)$ shows $m=2.7 \mathrm{~km}$ for O-trace and $m=7.7 \mathrm{~km}$ for the $\mathrm{E}_{s}$-trace. Thus, $P_{c}(t)$ calculated for $\mathrm{O}$ trace displayed better agreement with the $P_{e}(t)$. Both O- and $\mathrm{X}$-traces were again observed on the ionograms in the vicinity of $3.6 \mathrm{MHz}$ from 16:45 to 23:45 UT. The comparison of calculated phase paths with experimental ones showed better agreement for the $P_{c}(t)$ obtained for the ordinary trace. From
Fig. 3c it is evident that the discrepancy between $P_{c}(t)$ and $P_{e}(t)$ is considerably higher during the time intervals from 06:30 to 15:45 UT and 19:00 to about 23:00, during the periods when the $\mathrm{E}_{s}$ layer was present (examples of ionograms are presented in Fig. 7). The above finding is valid for all analysed time periods, when Es layer occurred in the vicinity of 3.6 MHz. Furthermore, Fig. 3c shows the phase paths obtained from manually corrected ionograms; manual correction removed outliers observed in uncorrected data (Fig. 3d) caused by automatic scaling. The average difference for autoscaled data was $m=9.4 \mathrm{~km}$, while after correction it decreased to $m=7.8 \mathrm{~km}$.

\subsection{Day 68 of 2004 (Fig. 3e)}

It was a quiet magnetic day: the $D_{s t}$ index was higher than $-6 \mathrm{nT}$. Again, to define $h_{0}$, we selected the time intervals, when only the O-trace was present close to $3.6 \mathrm{MHz}$. The $\mathrm{X}$-trace dominated on ionograms from 00:15 to 05:00 UT. 


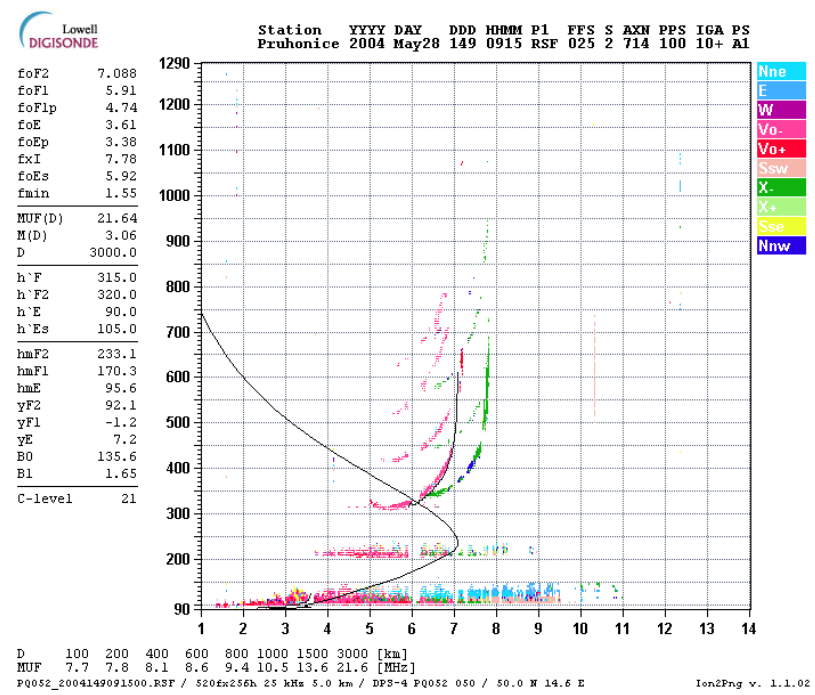

Fig. 5. An example of the ionogram recorded at Pruhonice observatory at 09:15 UT of the day 149 of 2004 when sporadic E was present. Black line shows the automatically scaled $N(h)$ profile by the ARTIST software.

During this time period $f \circ \mathrm{F} 2$ was less than $3.6 \mathrm{MHz}$ and we got no required digisonde data. The comparison of calculated phase paths with experimental ones for the time periods, when both $\mathrm{O}$ - and X-traces were present on the ionograms (e.g., from 05:15 to 06:30 UT), reveals $m=16.2 \mathrm{~km}$ and $m=4.3 \mathrm{~km}$ for $\mathrm{O}$ - and X-traces, respectively. Thus, $P_{c}(t)$ calculated for the X-trace agrees better with the $P_{e}(t)$. A similar situation was observed for the period from 15:30 to 23:45 UT. The comparison of $P_{c}(t)$ with $P_{e}(t)$ exhibits $m=14.1 \mathrm{~km}$ for O-trace and $m=5.4 \mathrm{~km}$ for X-trace in this interval. Thus, $P_{c}(t)$ calculated for $\mathrm{X}$-trace agrees better with the experimental $P_{e}(t)$. The discrepancy between $P_{c}(t)$ and $P_{e}(t)$, observed during the period from 06:45 to 16:00 UT, could be attributed partially to the presence of gaps on the ionograms just above $f o \mathrm{E}$ between the $\mathrm{E}$ and $\mathrm{F}$ layers, and partially to the affinity of $f o \mathrm{~F} 2$ layer to the working frequency of the Doppler apparatus. Examples of such ionograms are presented in Fig. 8. It is well known that regions of decreasing ionisation between the ionospheric layers do not reflect the radio waves and the resulting discontinuity leads to uncertainty in the true height above the valley region.

\subsection{Day 69 of 2004 (Fig. 3f)}

This day was one of the geomagnetically disturbed days $\left(D_{s t}\right.$ index started to decrease at noon and reached the minimum value of $-71 \mathrm{nT}$ at 23:00 UT). To define $h_{0}$, we selected the time intervals, when only the O-trace was present in the vicinity of 3.6 MHz. The X-trace of the ionospheric F2 layer dominated on the ionograms close to $3.6 \mathrm{MHz}$ from 04:00 to $05: 00 \mathrm{UT}$. As in the previous case, $f o \mathrm{~F} 2$ was less than $3.6 \mathrm{MHz}$ during this period, and we have got no required

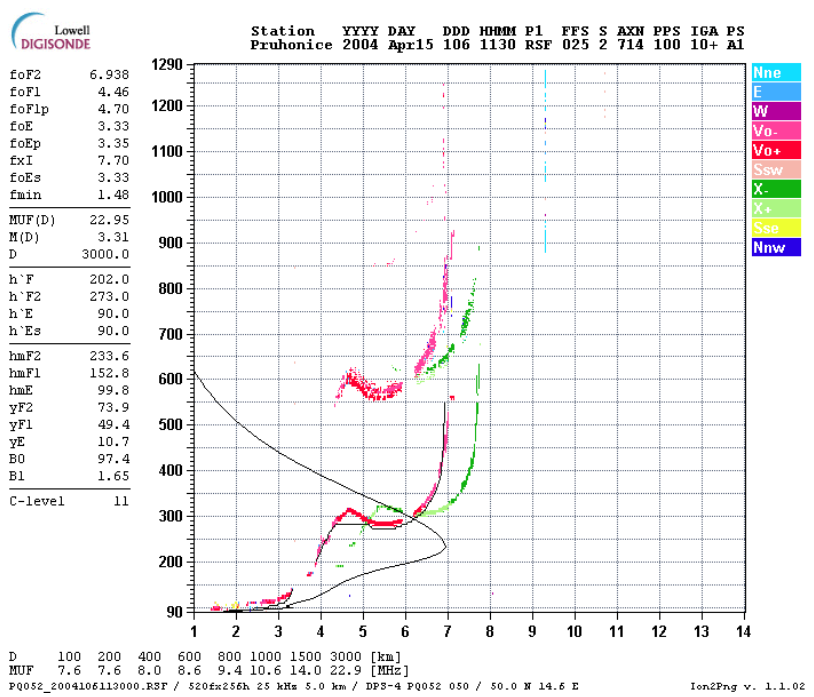

Fig. 6. An example of the ionogram recorded at Pruhonice observatory at 11:30 UT of the day 106, 2004, when O-trace was absent in the frequency range close to $3.6 \mathrm{MHz}$. Black line shows the automatically scaled $\mathrm{N}(\mathrm{h})$ profile.

digisonde data. Then we separated the time periods when both $\mathrm{O}$ - and $\mathrm{X}$-traces occurred on the ionograms nearby 3.6 MHz. After 22:15 UT, again, foF2 fell below $3.6 \mathrm{MHz}$, and only the X-trace was present on ionograms. That is why only $P_{e}(t)$ is plotted in the Fig. $3 \mathrm{f}$ after 22:15 UT. There is a "jump" upward (about $40 \mathrm{~km}$ ) of experimental and calculated phase paths from 18:00 to 18:15 (18:00 UT is shown by vertical dotted line in Fig. 3f). At this time $D_{s t}$ index dropped below $-50 \mathrm{nT}$.

We can also see large discrepancies between the phase paths (up to $40 \mathrm{~km}$ ) after 18:00 UT. This could mean a problem in ARTIST scaling (in its subprograms ARTNHPC used in ARTIST to invert scaled ionogram trace data into $\mathrm{N}(\mathrm{h})$ profiles working in real-time mode and JAVANHPC used in SAO Explorer) for geomagnetically disturbed ionosphere. Results of Chen et al. (1991, 1994) support this suggestion. They compared inverted $\mathrm{N}(\mathrm{h})$ profiles from digisonde ionograms recorded at Millstone Hill $\left(42.6^{\circ} \mathrm{N}, 284.5^{\circ} \mathrm{E}\right)$ and Arecibo $\left(18.5^{\circ} \mathrm{N}, 292 . .^{\circ} 9 \mathrm{E}\right)$ stations with those obtained from incoherent scatter radar measurements. They showed that the probable reason for the occurrence of larger deviations under the geomagnetic storm conditions is inaccuracy within the valley model.

Another example of the geomagnetically disturbed day is day 42 of 2004 (Fig. 4b). This strong wintertime storm started at about 09:00 UT on day 42 and achieved its maximum at 17:00 UT when the $D_{s t}$ index felt down to $-109 \mathrm{nT}$. Diurnal courses of the calculated and experimental phase paths obtained for the stormy day and for the preceding quite day of 10 February 2004 (day 41) are plotted in Figs. 4a and $\mathrm{b}$. An $\mathrm{X}$-trace was dominant during the night starting 

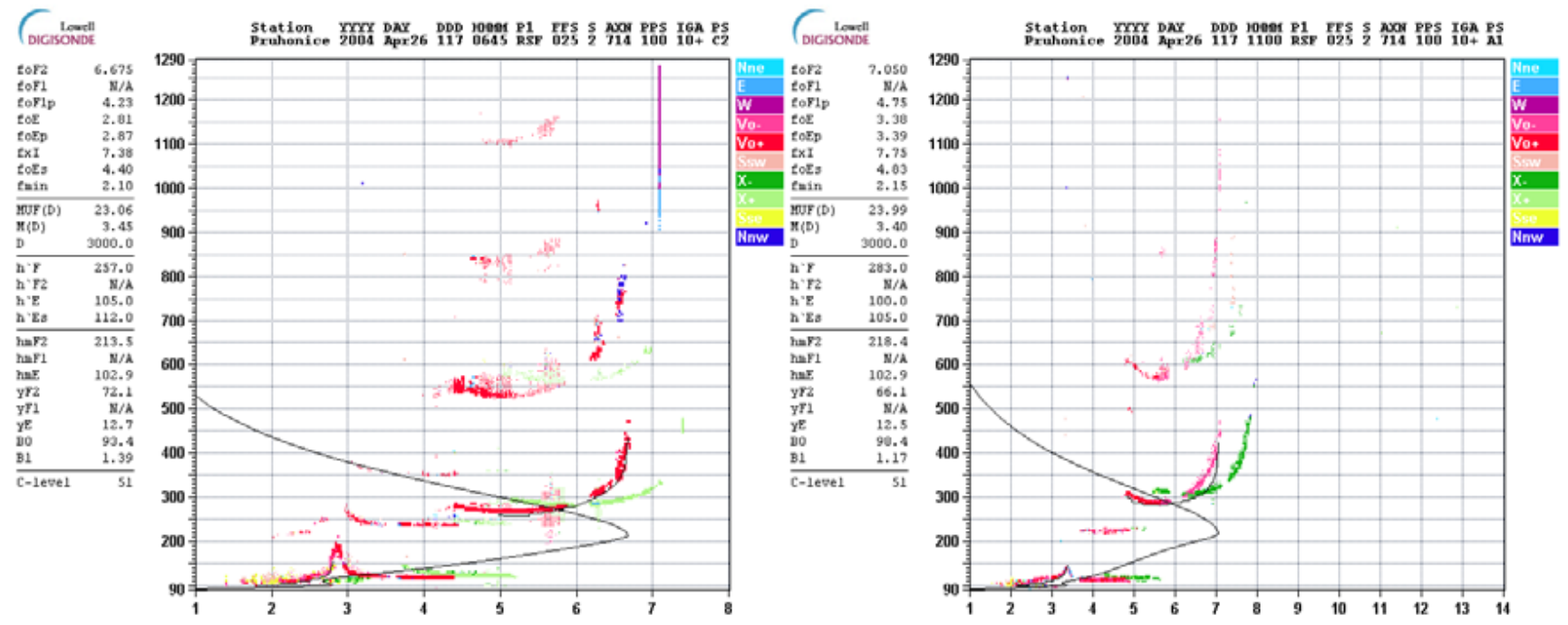

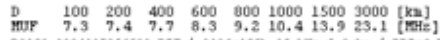

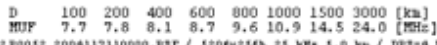

Fig. 7. Examples of ionograms recorded at Pruhonice observatory at 06:45 UT (left panel) and at 11:00 UT (right panel) for analysed day 117 of 2004, when sporadic E was present. Black line shows the automatically scaled N(h) profile.

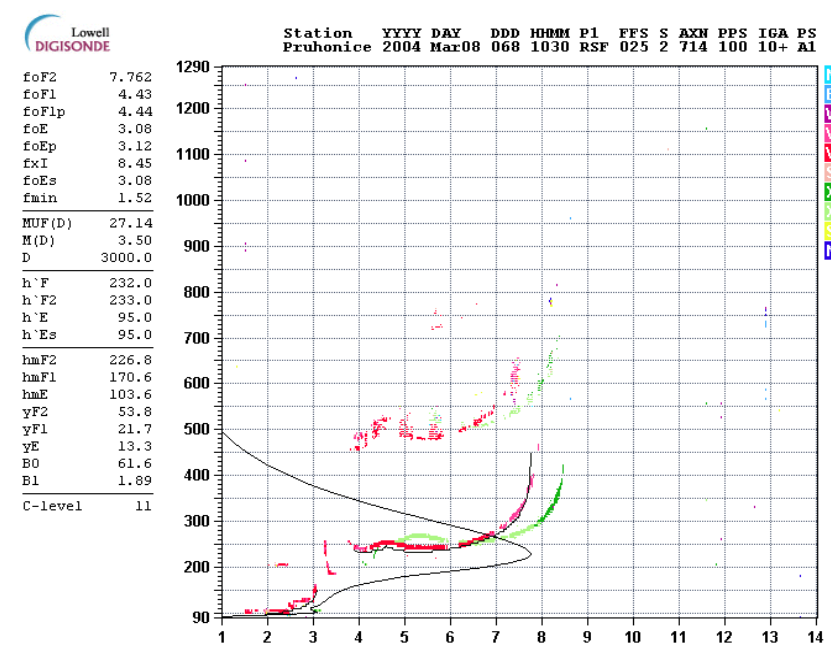

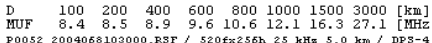

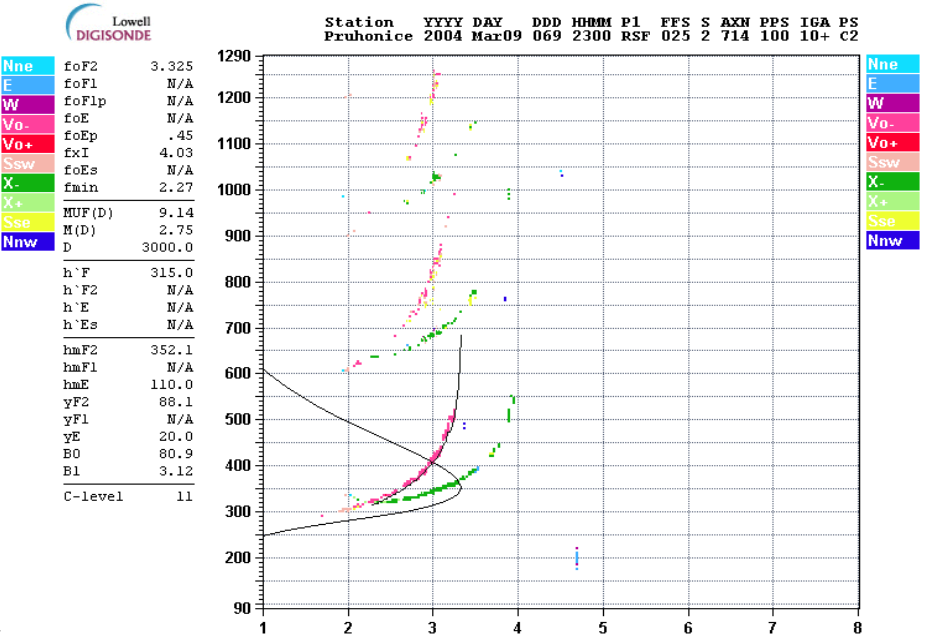

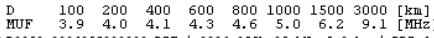

Fig. 8. Examples of the ionograms recorded at Pruhonice observatory during the day 68 of 2004: (a) ionospheric E-layer and gap between $\mathrm{E}$ and F layers; (b) affinity of critical frequency of F layer to the sounding frequency of the Doppler apparatus. Black line shows the automatically scaled $\mathrm{N}(\mathrm{h})$ profile. Time is in UT.

at about 17:00 UT till early morning hours (06:00 UT) during both 41 and 42 days. During several morning hours of day 41 and late evening hours of both analyzed days $f o \mathrm{~F} 2$ was below $3.6 \mathrm{MHz}$ and we have got no ionosondes data. Discrepancies between $P_{c}(t)$ and $P_{e}(t)$ observed within the period from about 07:00 UT to 16:00 UT on day 41 could be attributed to the presence of gaps on the ionograms just above foE between $\mathrm{E}$ and $\mathrm{F}$ layers. The gap in the diurnal course of $P_{e}(t)$ from 08:00 till 15:00 UT on day 42 is due to a low quality of Doppler records. Significant differences between the both $P_{c}(t)$ and $P_{e}(t)$ phase paths appeared after 15:00 UT when $D_{s t}$ dropped below $-50 \mathrm{nT}$ (this time is shown by vertical dotted line).

Day 96 of 2004 (Fig. 4d) was also a disturbed day. A moderate geomagnetic storm had its onset at about 08:00 UT. Between 17:00 UT and 18:00 UT the $D_{s t}$ became lower than $-50 \mathrm{nT}$. The storm culminated near 19:00 UT when the $D_{s t}$ index reached maximum of $-81 \mathrm{nT}$. Phase paths calculated 
from Doppler and digisonde records for the disturbed day 96 are compared with those obtained for quiet day 93 in Figs. 4c and d. During both 93 and 96 days the X-trace dominated during the night starting at about 19:00 UT. A sporadic Elayer was present on the ionograms during the day 93 from 11:00 to 12:15 UT. An appearance of the Es could be considered as a cause of difference between $P_{c}(t)$ and $P_{e}(t)$ within this time period. We have got no data till 04:45 UT for the stormy day because of low foF2. Beginning of the considerable discrepancies between phase paths (up to $37 \mathrm{~km}$ ) we observed after 18:30 UT under storm conditions.

The fact that ARTIST is not very reliable under certain conditions (as any other autoscaling program) is well-known fact, at least in the European ionosonde community (e.g., Zolesi et al., 2004) in spite of the fact that ARTIST underwent several times modifications that improved the quality of its analysis and its network-wide upgrade is underway (Galkin et al., 2006). For this reason the European ionospheric project COST296 runs database of automatically scaled results (parameters, profiles), and separately database of manually checked results (parameters, profiles). Main problems appear at $\mathrm{F} 1$ region heights, maybe as a consequence of the E-F region valley influence. Our Doppler sounding frequency is very often reflected just at F1 region heights.

The days shown here are typical examples of the behaviour of the difference between the phase paths computed from ionograms and from the Doppler system measurements. We analysed more days, but we cannot present in one paper all days, this would make the paper long and boring.

We have been working with a frequency of $3.6 \mathrm{MHz}$, which is during daytime usually well below foF2. This means that we can often use Doppler system measurements to improve ionogram inversion into the electron density profile at heights well below the $\mathrm{F} 2$ region maximum and, thus, the effect of possible differences, formed in the height interval between the $3.6 \mathrm{MHz}$ reflection height and $h m \mathrm{~F} 2$, on the $h m \mathrm{~F} 2$ determination is not included.

\section{Conclusions}

The comparison of calculated $P_{c}(t)$ and experimental $P_{e}(t)$ phase paths for 21 selected days of 2004 allows us to estimate the magnitude of the difference between the values of the phase path obtained by the Digisonde Portable Sounder DPS4 and the new Doppler type system during the common volume measurements at European midlatitude station Pruhonice. To avoid additional inaccuracy, scaling of all ionograms obtained from DPS-4 was manually corrected. It is also important to note that days of 2004 involved in the analysis were geomagnetically quiet days, except for days 42, 69 and 96, when moderate-to-intense storm conditions took place. The overall average difference between $P_{c}(t)$ and $P_{e}(t)$ is $m=11.1 \pm 1.2 \mathrm{~km}$, standard deviation is $5.0 \mathrm{~km}$. In the best case the average daily difference was about $3 \mathrm{~km}$, which is comparable with the height resolution of the digisonde measurements. The substantial disagreement between $P_{c}(t)$ and $P_{e}(t)$ is obtained in three cases: (1) presence of $\mathrm{E}_{s}$ layer, (2) when the sounding frequency is close to the critical frequency of ionospheric layer, and (3) during geomagnetic storms (see Figs. 3f, 4b and d). On the other hand, during geomagnetically quiet days, absence of the sporadic E layer, and at availability of high quality ionograms and correct scaling, the electron density profiles, calculated by the Automatic Real Time Ionogram Scaler with True height algorithm (ARTIST) on the basis of such ionograms, can be considered reliable. Obviously the comparison of experimental and calculated phase paths can help to test the inversion codes of electron density profiles.

Acknowledgements. Authors acknowledge support by the grant No. 205/04/2110 of the Grant Agency of the Czech Republic. Authors thank both referees for their helpful comments.

Topical Editor M. Pinnock thanks L.-A. McKinnell and another referee for their help in evaluating this paper.

\section{References}

Bibl, K.: Evolution of ionosonde, Annali di Geophys., 41(5-6), 667-680, 1998.

Bossy, L.: Accuracy comparison of ionogram inversion methods, Adv. Space Res., 14(12), 39-42, 1994.

Chen, C.-F., Ward, B. D., Reinisch, B. W., and Buonsanto, M. J.: Ionosonde observations of the E-F valley and comparison with incoherent scatter radar profiles, Adv. Space Res., 11(10), 8992, 1991.

Chen, C.-F., Reinisch, B. W., Scali, J. L., and Huang, X.: The accuracy of ionogram-derived N(h) profiles, Adv. Space Res., 14(12), 43-46, 1994.

Davies, K.: Ionospheric radio waves, Blaisdell Publishing Company. A Division of Ginn and Company, Waltham, Massachusetts - Toronto - London, 1969.

Galkin, I. A. and Dvinskikh, N. I.: Interpretation of vertical incidence sounding data by electronic computing machine BECM2M (in Russian), Studies Geomagn. Aeronautic Phys., Sun 3 (part 1), 109-143, 1968.

Galkin, I. A., Khmyrov, G. M., Kozlov, A., Reinisch, B. W., Huang, X., and Kitrosser, D. F.: Ionosonde networking, databasing, and web serving, Radio Sci., 41(5), RS5S33, doi:10.1029/2005RS003384, 2006.

Paul, A. K.: Limitations and possible improvements of ionospheric models for radio propagation: Effects of sporadic E layers, Radio Sci., 21(3), 304-308, 1986.

Pezzopane, M. and Scotto, C.: The INGV software for the automatic scaling of foF2 and MUF(3000)F2 from ionograms: A performance comparison with ARTIST 4.01 from Roma data, J. Atmos. Sol.-Terr. Phys., 67, 1063-1073, 2005.

Reinisch, B. W. and Huang, X.: Automatic calculation of electron density profiles from digital ionograms, 3 , Processing of botomside ionograms, Radio Sci, 18, 477-492,1983.

Reinisch, B. W., Huang, X., Galkin, I. A., Paznukhov, V., and Kozlov, A.: Recent advances in real-time analysis of ionograms and 
ionospheric drift measurements with digisondes, J. Atmos. Sol.Terr. Phys., 67, 1054-1062, 2005.

Titheridge, J. E.: Starting models for the real height analysis of ionograms, J. Atmos. Sol.-Terr. Phys., 48, 435-446, 1986.

Wright, J. W., Laird, A. R., Obitts, D., Violette, E. J., and McKinnis, D.: Automatic $\mathrm{N}(\mathrm{h}, \mathrm{t})$ profiles of the ionosphere with a digital ionosonde, Radio Sci., 7, 1033-1043, 1972.
Zolesi, B., Belehaki, A., Tsagouri, I., and Cander, L. R.: Real-time updating of the Simplified Ionospheric Regional Model for operational applications, Radio Sci., 39, RS2011, doi:10.1029/2003RS002936, 2004. 